Original Paper http://ajol.info/index.php/ijbcs http://indexmedicus.afro.who.int

\title{
Modèle de distribution des nématodes chez le poisson chat (Clarias anguillaris (Linnaeus, 1758)) dans deux réservoirs du Burkina Faso (Afrique de l'Ouest)
}

\author{
Yamba SINARE ${ }^{1,2^{*}}$, Magloire BOUNGOU ${ }^{1}$, Malika N. KANGOYE ${ }^{1}$ et \\ Gustave B. KABRE ${ }^{1}$ \\ ${ }^{I}$ Laboratoire de Biologie et Ecologie Animales, Département de Biologie et Physiologie Animales, \\ Université Joseph Ki-Zerbo, 03 BP 7021, Ouagadougou, Burkina Faso. \\ ${ }^{2}$ Institut Des Sciences, 01 BP 1757, Ouagadougou, Burkina Faso. \\ *Auteur correspondant ; E-mail : sinareyamba@yahoo.fr; Tel. : (00226)76157990
}

Received: 30-11-2020

Accepted: 07-06-2021

Published: 30-06-2021

\section{RESUME}

Clarias anguillaris est un poisson très consommé au Burkina Faso. Parmi les parasites de ce poisson, on note les nématodes dont la diversité et les organes de prédilection ne sont pas très bien connus. Ce travail montre la diversité et la distribution des nématodes chez $C$. anguillaris. Les poissons hôtes ont été échantillonnés dans deux réservoirs du Burkina Faso : le réservoir de Loumbila et celui de Ziga. À chaque échantillonnage, les poissons hôtes ont été examinés à la recherche des nématodes. Le tube digestif a été séparé des organes annexes après dissection, et chaque partie a été examinée, pour la récolte des nématodes. Au total, 268 Clarias anguillaris ont été examinés. 74,63\% des poissons examinés étaient infestés. 06 espèces de nématodes ont été récoltées. Il s'agit de Paracamallanus cyathopharynx, Procamallanus laevichoncus, Rhabdochona congolensis, Contracaecum sp., Anisakis sp. et une espèce non identifiée. Les nématodes étaient plus nombreux dans le réservoir de Ziga. Cependant, la prévalence des nématodes n'a pas varié dans les deux sites. Les nématodes adultes étaient plus diversifiés dans le tube digestif et les larves étaient fréquentes dans la cavité et dans les tissus des organes. Parmi les espèces de nématodes étudiés, Anisakis sp. a été rencontrée chez les poissons pour la première fois au Burkina Faso. Cette investigation a permis de connaître les espèces de nématodes qu'héberge $C$. anguillaris au Burkina Faso. Ces espèces n'avaient pas la même distribution chez l'hôte de même que les différents stades. Les adultes étaient rencontrés dans le tube digestif et les larves dans les tissus et les mésentères.

(C) 2021 International Formulae Group. All rights reserved.

Mots clés : Poisson, parasites, helminthes, Ziga, Loumbila.

\section{Nematode distribution pattern in catfish (Clarias anguillaris (Linnaeus, 1758)) in two reservoirs of Burkina Faso (West Africa)}

\begin{abstract}
Clarias anguillaris is a fish widely consumed in Burkina Faso. Among the parasites of this fish, we note the nematodes whose diversity and preferred organs are not very well known. This work shows diversity and distribution of nematodes of $C$. anguillaris. The host fish were sampled in two reservoirs in
\end{abstract}


Burkina Faso; namely the Loumbila dam and the Ziga dam. At each sampling, the host fish were examined for nematodes. The digestive tract, for its part, was separated from the secondary organs after dissection, and each part was examined for the collection of nematodes. A total of 268 Clarias anguillaris were examined. Of the fish examined, $74.63 \%$ were infected. 06 species of Nematodes were collected: Paracamallanus cyathopharynx, Procamallanus laevichoncus, Rhabdochona congolensis, Contracaecum sp., Anisakis sp. and an unidentified nematode. Nematodes were more abundant in Ziga dam. However, the percentage of nematodes infestation did not vary at both sites. Adults' nematodes were more diverse in the digestive tract and larva were more found in the cavity and in the tissues. Among the species of nematodes studied, Anisakis sp. was encountered for the first time on fishes in Burkina Faso. This investigation allowed to discover the nematode species harbored by $C$. anguillaris in Burkina Faso. These species did not have the same distribution in the host nor the different stages of the nematodes. Adults were found in the digestive tract and larvae in tissues and mesenteries.

(C) 2021 International Formulae Group. All rights reserved.

Keywords: Fish, parasites, helminths, Ziga, Loumbila.

\section{INTRODUCTION}

Les parasites font partie intégrante de l'évolution naturelle des animaux et ils représentent un intérêt en soi (Mclaughlin and Concordia, 2008). Selon Kuchta et al. (2012), 359 espèces d'helminthes ont été reportées chez les poissons en Afrique. Les poissons affectés deviennent inaptes, perdent du poids et les mortalités se produisent dans les cas d'infestations lourdes en particulier avec les vibrioses secondaires (Chong et al., 2011). Selon FAO/WHO (2014), certains parasites de poissons sont responsables de zoonoses.

Les nématodes affectent beaucoup d'espèces de poissons d'élevage et de poissons sauvages (Gibbons, 2010). Clarias anguillaris (Linnaeus, 1758) est un poisson prisé par la population du Burkina Faso et peut être utilisé en élevage (Compaoré et al., 2016). Il est consommé frais, fumé ou frit. Pourtant, peu d'études se sont intéressées à la pathologie de ce poisson dans ce pays (Sinaré, 2009 ; Sinaré et al., 2015; Sinaré, 2017). Généralement, les nématodes ne provoquent pas de pathologies très graves pouvant conduire à la mort du poisson; mais ils occasionnent d'autres infections causées par les virus et les bactéries (Usip et al., 2010). Les nématodes qui possèdent des organes d'ancrage (comme Paracamallanus cyathopharynx) créent des nodules et des entérites au lieu d'attachement des piquants (Akinsanya and Otubanjo, 2006). Ils peuvent entrainer un amaigrissement des poissons et en résulter une perte économique. Les poissons s'infestent en consommant de la nourriture souillée contenant des stades infectants ou en cohabitant avec des animaux qui permettent aux nématodes de compléter leur cycle de vie (par exemple les hôtes intermédiaires). Le but de cette étude était d'inventorier les nématodes inféodés aux poissons $C$. anguillaris et de comprendre leur distribution chez l'hôte.

\section{MATERIEL ET METHODES \\ Sites d'étude}

La présente étude a été conduite sur deux principaux réservoirs non loin de Ouagadougou, ville de près de 1.500 .000 habitants et capitale politique du Burkina Faso : le réservoir de Loumbila et celui de Ziga (Figure 1). Ces deux réservoirs se situent sur le fleuve Nakanbé en zone soudanosahélienne.

Le réservoir de Loumbila $\left(12^{\circ} 29^{\prime} \mathrm{N}\right.$, $\left.01^{\circ} 24^{\prime}, W\right)$ se situe à une vingtaine de kilomètres au Nord Est de Ouagadougou. Il a été construit en 1947 et possède une capacité d'environ 42 millions de mètre cube $\left(\mathrm{m}^{3}\right)$. La profondeur moyenne de ce lac est de $2,15 \mathrm{~m}$ et sa superficie en pleine eau est de $16,80 \mathrm{~km}^{2}$. 
Les activités socio-économiques de la population riveraine sont essentiellement l'agriculture, l'élevage, la pêche et le commerce.

Le réservoir de Ziga $\left(12^{\circ} 37^{\prime} 03.22^{\prime \prime}\right.$ $\mathrm{N}$ et $0^{\circ} 49^{\prime} 23.43^{\prime \prime}$ W) est situé à l'Est de Ouagadougou. Il a une capacité de 200 millions de $\mathrm{m}^{3}$ et s'étend sur $70 \mathrm{Km}^{2}$. Le lac de barrage de Ziga a été construit en 2000. Les populations riveraines du lac pratiquent le maraîchage sur les rives après la digue.

\section{Collecte et traitement des échantillons}

L'échantillonnage des poissons a eu lieu de janvier 2011 à septembre 2012. Les poissons ont été collectés directement sur les sites pendant la saison sèche et la saison pluvieuse. Les spécimens échantillonnés proviennent de la pêche commerciale. Dès le débarquement des prises par les pêcheurs, des spécimens vivants de $C$. anguillaris de tailles différentes ont été prélevés chez les pêcheurs. Ces spécimens toujours vivants ont été transportés au Laboratoire dans un bac contenant de l'eau du réservoir.

Au laboratoire, chaque spécimen a été formellement identifié et pesé au gramme près à l'aide d'une balance. La longueur standard de chaque spécimen a été mesurée au millimètre près, à l'aide d'un ichtyomètre. Une fois les mensurations terminées, les poissons ont été disséqués à l'aide de ciseaux et de pinces. Après dissection, la détermination du sexe de chaque poisson a été faite par simple observation à l'œil nu de l'appareil reproducteur. La taille des gonades a été mesurée avec l'ichtyomètre et leur poids à l'aide d'une balance électronique sensible à $1 / 1000$ de gramme près. Les différents organes (estomac, intestin, organes annexes, rein, muscle, branchies, gonades) ont été placés dans des boites de Pétri en vue de récolter les parasites. Les parasites ont été également recherchés dans la cavité générale.

Les parasites prélevés ont été placés d'abord dans une boite de Pétri contenant de l'eau saline. Ils ont été ensuite montés frais au microscope entre lame et lamelle pour l'observation. L'identification des nématodes a été faite en utilisant les documents de Gibbons (2010), Kabré (1997) et Yamaguti (1961). Après observation, les spécimens ont été conservés dans de l'alcool $70^{\circ}$.

\section{Analyse des données}

Pour les analyses statistiques, le logiciel Statview 5.0.1.0 version SAS Institute Inc a été utilisé. La dynamique et la distribution des nématodes a été analysée en fonction des paramètres biotiques (taille, poids, sexe, organes infestés) et abiotiques (site, saison). Le test non paramétrique de Mann Whitney a été utilisé pour analyser l'importance du facteur site et du facteur saison. Le sexe a aussi été testé comme facteur pouvant affecter le parasitisme en utilisant toujours le même test. Pour confirmer l'idée selon laquelle les parasites ont une certaine prédilection pour certains organes, nous avons utilisé le test non paramétrique de Kruskal Wallis. Les liens entre le parasitisme et les variables taille et poids de l'hôte ont été testés en utilisant la régression linéaire. Le seuil de signification était fixé à $5 \%$. 


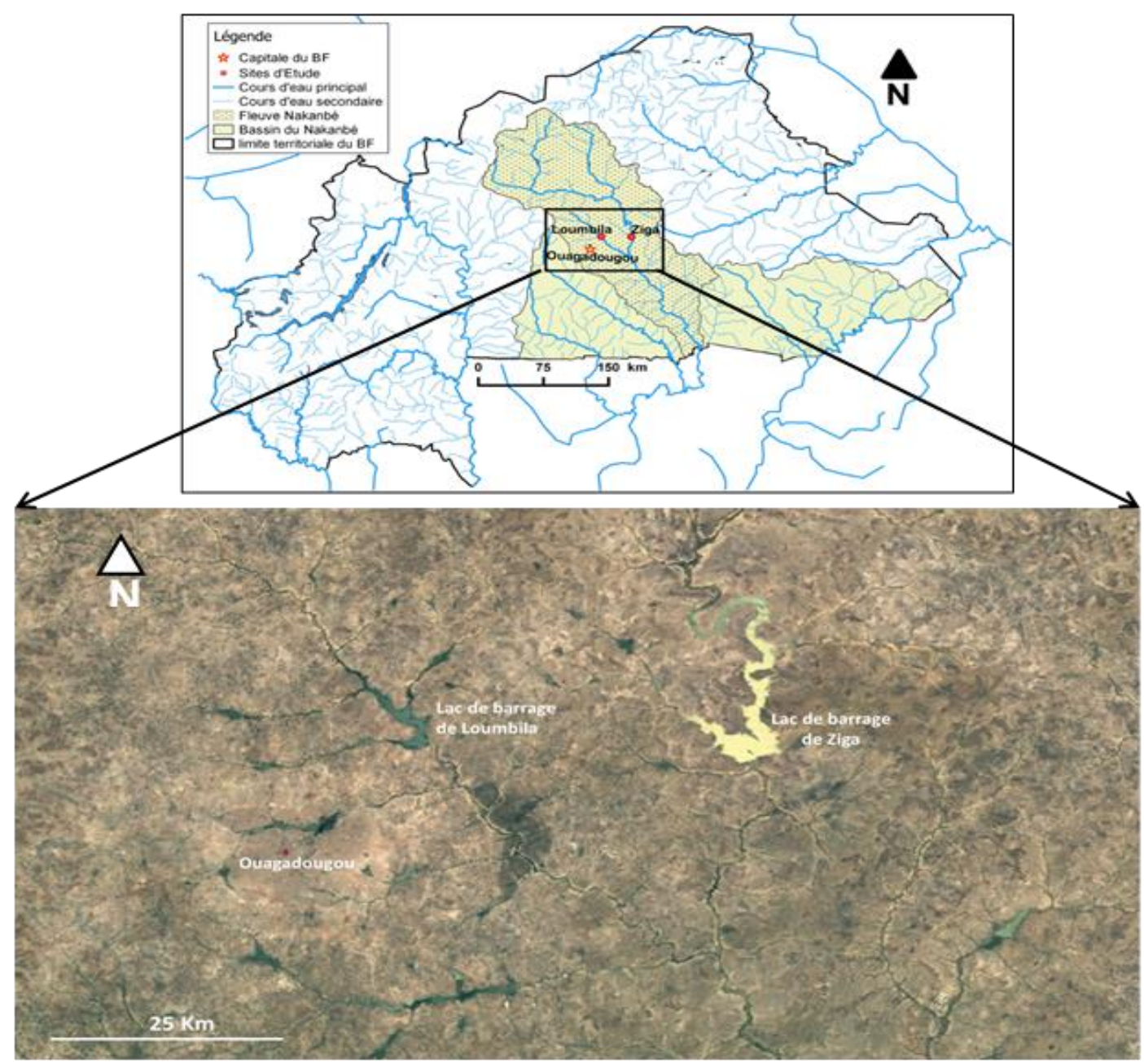

Figure 1 : Carte présentant les sites d'étude (google map modifié).

\section{RESULTATS}

Diversité des nématodes chez Clarias anguillaris

Sur 268 spécimens de Clarias anguillaris examinés, $74,63 \%$ ont été parasités. Un total de 06 espèces de nématodes a été inventorié. Il s'agit de Paracamallanus cyathopharynx, (Baylis, 1923), Procamallanus laevichoncus (Weld, 1862), Rhabdochona congolensis CampanaRouget, 1961, Contracaecum sp, Anisakis sp. et un nématode non identifié. Anisakis sp a été rencontré pour la première fois chez les poissons au Burkina Faso. P. cyathopharynx, $P$. laevichoncus et le nématode non identifié étaient des parasites adultes. Contracaecum $\mathrm{sp}$ et Anisakis sp. étaient des parasites au stade larvaire. $R$. congolensis a été observés sous les deux formes (larvaire et adulte).

\section{Distribution des nématodes chez Clarias anguillaris}

Le Tableau 1 présente le pourcentage d'infestation et l'intensité des nématodes inventoriés en fonction de leurs sites de fixation. L'analyse des résultats de ce tableau montre une différence significative du pourcentage de l'infestation entre les organes de fixation des nématodes chez $C$. anguillaris. En effet, le test de comparaison de Kruskall Wallis a montré une différence significative entre le nombre de parasites et leurs 
localisations $(\mathrm{p}=0.0001)$. L'organe le plus infesté par les nématodes était l'intestin avec un pourcentage d'infestation de $52,24 \%$ et une abondance totale de 712 nématodes. Il était suivi de la cavité qui présentait un pourcentage de $25,37 \%$ et une abondance totale de 518 nématodes. Pour ce qui est de l'intensité parasitaire, la paroi de l'intestin était la plus parasitée avec une intensité de 31,09 et une abondance totale de 1057 nématodes. Il était suivi de la paroi de l'estomac qui présentait une intensité de 24,43 et une abondance totale de 171 nématodes.

Le Tableau 2 présente la distribution des espèces de nématodes dans les différents organes de $C$. anguillaris. Certaines espèces de nématodes peuvent envahir plusieurs organes, de même qu'un organe peut contenir plusieurs espèces. Ainsi, 02 espèces étaient présentes dans le foie, 05 dans l'estomac (02 dans la paroi) et 05 dans l'intestin (02 dans la paroi) donnant un total de 06 espèces dans le tube digestif. 03 espèces étaient présentes dans la cavité générale.

Rhabdochona congolensis et Anisakis sp sont les deux nématodes rencontrés dans tous les organes observés.

Rhabdochona congolensis était rencontré dans la paroi de l'estomac et de l'intestin, dans le foie et dans la cavité générale sous forme larvaire. Les larves étaient plus abondantes dans la paroi de l'estomac. C'était le parasite le plus abondant rencontré chez Clarias anguillaris. Les adultes de $R$. congolensis ont été rencontrés uniquement à l'intérieur de l'intestin.

Anisakis sp était l'espèce la plus abondante dans la cavité générale et dans le foie. Elle était rencontrée généralement sur les mésentères de la cavité. Elle a été également rencontrée dans d'autres organes (estomac et intestin) mais à très faible densité par rapport à la cavité.

Procamallanus laeviconchus, Paracamallanus cyathopharynx et Contracaecum sp ont été rencontrés dans deux organes.

Procamallanus laeviconchus était l'espèce la plus présente dans l'estomac.
Quelques spécimens de P. laeviconchus ont été dénombrés dans l'intestin.

Tout comme $P$. laeviconchus, $P$. cyathopharynx a été observé dans l'intestin et dans l'estomac. Mais contrairement à $P$. laeviconchus, $P$. cyathopharynx était plus abondant et plus fréquent dans l'intestin. Les spécimens de $P$. cyathopharynx étaient généralement rencontrés au niveau de l'intestin postérieur vers le rectum; certains se présentaient même au niveau de l'orifice anal.

Contracaecum sp était présent dans l'intestin mais contrairement aux deux autres nématodes $(P$. laeviconchus et $P$. cyathopharynx), il était également présent dans la cavité générale. Dans la cavité générale, il était localisé sur les mésentères sous forme de larves encapsulées et dans la paroi de l'intestin sous forme de larves enkystées. Les spécimens étaient plus abondants et plus fréquents dans la cavité.

Le nématode non identifié était un oxyure adulte rencontré uniquement au niveau de l'estomac. Les spécimens observés étaient tous des femelles. Leur utérus contenait des œufs embryonnés.

L'estomac était plus chargé de nématodes par rapport aux autres organes à cause des larves très abondantes dans sa paroi. Il était suivi de l'intestin et de la cavité générale; l'organe infecté le moins chargé de nématodes étant le foie. Les autres organes comme les branchies, les gonades, les reins et les muscles n'ont pas été infectés par les nématodes dans cette étude. Les nématodes adultes ont été rencontrés dans la lumière du tube digestif (estomac et intestin) et les larves ont été trouvées encapsulées ou enkystées soit dans la cavité générale, soit sur les mésentères ou dans les tissus des organes.

\section{Dynamique et distribution des nématodes en fonction des paramètres biotiques (taille, poids, sexe)}

La distribution des nématodes en fonction de la taille des hôtes a été étudiée en regroupant les hôtes par classe de taille. Les différents indices de l'infestation en fonction de la taille sont présentés dans la Figure 2. Cette figure montre que le plus haut 
pourcentage se situait chez les spécimens dont la taille était comprise entre 31,6 et $41,6 \mathrm{~cm}$ (Figure 2A). Le pourcentage a été croissant jusqu'à cet intervalle de taille et décroit à partir de celui-ci jusqu'à $61,6 \mathrm{~cm}$. Avec les courbes de l'abondance et de l'intensité moyennes (Figure 2B), nous avons constaté que l'intensité et l'abondance des nématodes augmentaient avec la taille des poissons hôtes. Cependant, l'intensité et l'abondance étaient basses entre 41,6 et 51,6 cm. Au niveau des adultes, on constatait que l'intensité augmentait pendant que le pourcentage de l'infestation diminuait. Cela signifie que les plus grands poissons étaient moins infestés et plus chargés. Il n'y avait pas de corrélation entre le nombre de nématodes et la taille individuelle des hôtes d'une part et d'autre part entre le nombre de nématodes et le poids des gonades des spécimens hôtes. Cependant, le nombre des larves de Rhabdochona congolensis était corrélée avec la taille individuelle des hôtes $(\mathrm{p}=0,03)$ et le nombre de Anisakis sp était corrélée avec la taille des gonades $(\mathrm{p}=0,04)$. Le nombre des larves augmentait avec la taille des hôtes et des gonades.

Il n'y avait pas de différence significative entre le nombre de nématodes rencontrés et le sexe du poisson. Les poissons mâles pouvaient contenir ou être infestés d'autant de nématodes que les femelles.

\section{Dynamique et distribution des nématodes en fonction des paramètres abiotiques (site, saison)}

La Figure 3 nous présente la prévalence et l'intensité moyenne des espèces de nématodes en fonction des saisons. Cette figure montre que la prévalence des espèces était plus élevée en saison pluvieuse sauf chez Anisakis sp où la prévalence était plus élevée en saison sèche. Ce qui signifie que pendant la saison sèche, les poissons sont plus infestés par Anisakis sp ou sont plus en contact avec lui. La saison sèche favorise son infection. Pour les autres espèces, la saison pluvieuse est un facteur qui permet leur transmission aux poissons hôtes. La saison pluvieuse augmente le taux d'infestation des hôtes. $\mathrm{La}$ comparaison du nombre de nématodes entre les saisons montrait que les nématodes étaient plus abondants en saison sèche. Cette différence était statistiquement significative ( $\mathrm{p}$ $=0,0430$, test $\mathrm{U}$ de Mann Whitney). Les spécimens de $P$. cyathopharynx avaient la plus haute prévalence dans toutes les saisons avec une prédominance en saison pluvieuse. C'était également l'espèce qui montrait une prévalence élevée dans les deux sites (Figure 4).

L'intensité des Rhabdochona congolensis était la plus dominante. L'intensité de Contracaecum sp, $P$. cyathopharynx et $P$. laeviconchus était plus haute en saison pluvieuse et celle de Anisakis sp et Rhabdochona congolensis en saison sèche. La différence était statistiquement significative pour Rhabdochona congolensis $(\mathrm{p}=0,04)$ et $P$. laeviconchus $(\mathrm{p}=0,01)$.

La Figure 4 nous présente la prévalence et l'intensité moyenne des espèces de nématodes en fonction des sites d'étude. La différence du nombre de nématodes entre les deux sites était significative $(p=0,0286)$. Le nombre de nématodes parasites des spécimens de $C$. anguillaris était plus important dans le réservoir de Ziga que dans celui de Loumbila.

En termes de diversité spécifique, le réservoir de Ziga présentait autant d'espèces de nématodes que celui de Loumbila. Pour les espèces communes aux deux sites, la prévalence de $P$. laeviconchus, $P$. cyathopharynx et de Anisakis sp était plus élevée dans le réservoir de Loumbila que dans celui de Ziga ; la prévalence de Contracaecum sp. et de $R$. congolensis était plus élevée dans le réservoir de Ziga que dans celui de Loumbila. Contracaecum sp, Anisakis sp et $R$. congolensis présentaient des intensités très fortes dans le réservoir de Ziga; par contre l'intensité de $P$. laeviconchus était plus élevée dans le réservoir de Loumbila $(\mathrm{p}=0,01)$. L'intensité de $P$. cyathopharynx n'avait presque pas varié dans les deux sites. Les larves de nématodes rencontrées dans la paroi du tube digestif ne s'étaient rencontrées que chez les poissons du réservoir de Ziga et elles étaient plus nombreuses en saison sèche. 
Tableau 1 : Pourcentage et intensité des Nématodes observés en fonction de leurs sites de fixation.

\begin{tabular}{lcccc}
\hline Organes infectés & $\begin{array}{c}\text { Nombre de } \\
\text { poissons infectés }\end{array}$ & Taux d'infestation (\%) & $\begin{array}{c}\text { Nombre de } \\
\text { parasites }\end{array}$ & Intensité \\
\hline Cavité & 68 & 25,37 & 518 & 7,62 \\
Estomac & 61 & 22,76 & 231 & 3,79 \\
Foie & 6 & 2,24 & 17 & 2,83 \\
Intestin & 140 & 52,24 & 712 & 5,09 \\
Paroi estomac & 34 & 12,69 & 1057 & 31,09 \\
Paroi intestin & 7 & 2,61 & 171 & 24,43 \\
\hline
\end{tabular}

Tableau 2 : Prévalence et abondance (entre parenthèse) des espèces de Nématodes dans les différents organes infestés.

\begin{tabular}{|c|c|c|c|c|c|}
\hline Espèces & $\begin{array}{l}\text { Prévalence \% } \\
\text { (abondance) }\end{array}$ & Cavité & Estomac & Foie & Intestin \\
\hline Contracaecum sp & $17,54(290)$ & 14,55 (199) & - & - & 2,99 (91) \\
\hline Anisakis sp & $14,55(308)$ & 11,57 (290) & $1,12(4)$ & $1,49(12)$ & $0,37(2)$ \\
\hline P. cyathopharynx & 50,37 (604) & - & $0,75(2)$ & - & $49,63(602)$ \\
\hline P. laeviconchus & $21,64(182)$ & - & $20,52(171)$ & - & $1,12(11)$ \\
\hline R. congolensis & 24,63 (1316) & $1,12(29)$ & $14,18(1105)$ & $0,75(5)$ & $8,58(177)$ \\
\hline $\begin{array}{l}\text { Nématode non } \\
\text { identifié. }\end{array}$ & $0,37(6)$ & - & $0,37(6)$ & - & - \\
\hline
\end{tabular}



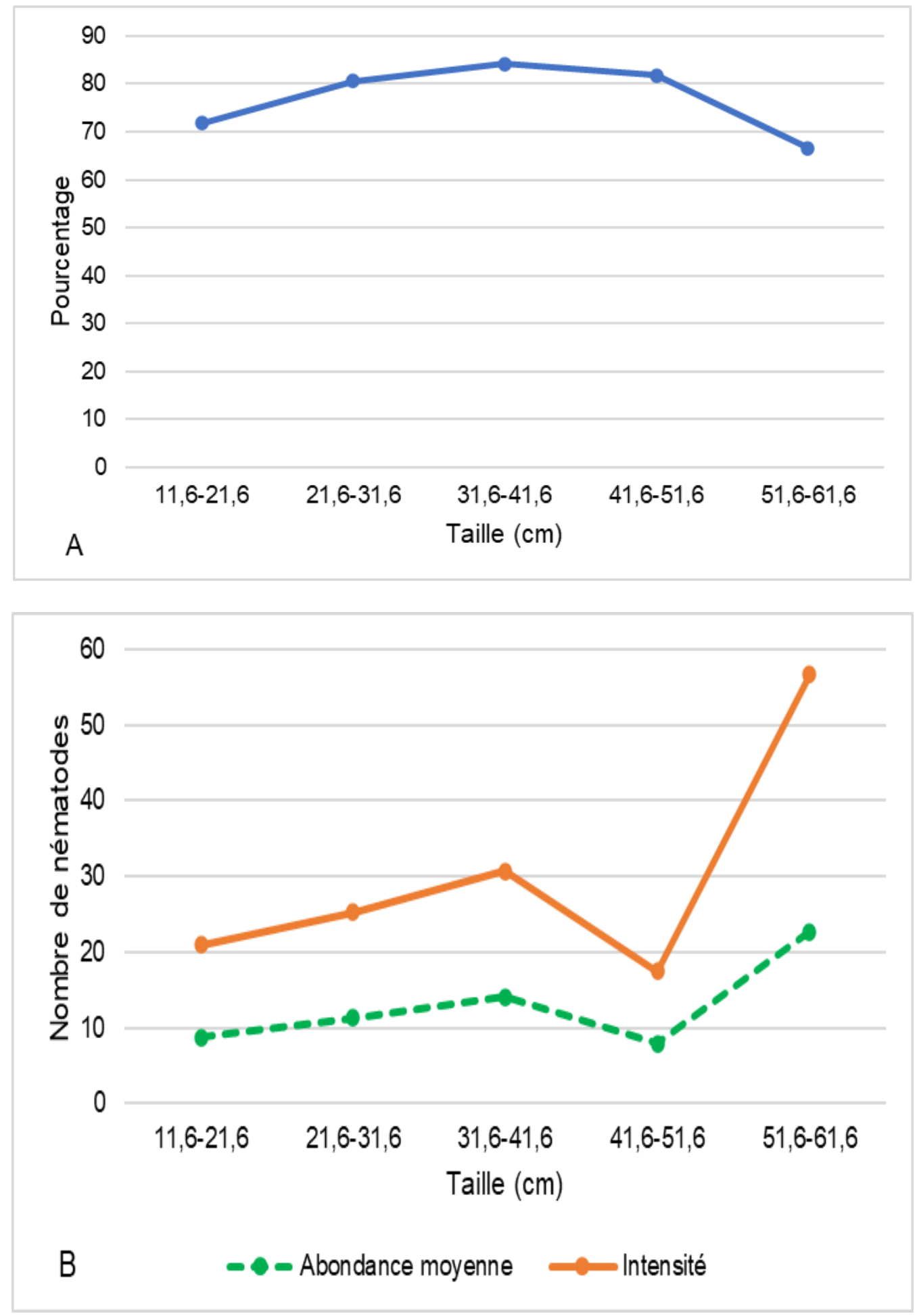

Figure 2 : Pourcentage (A), abondance et intensité moyennes (B) des nématodes en fonction de la taille des hôtes. 

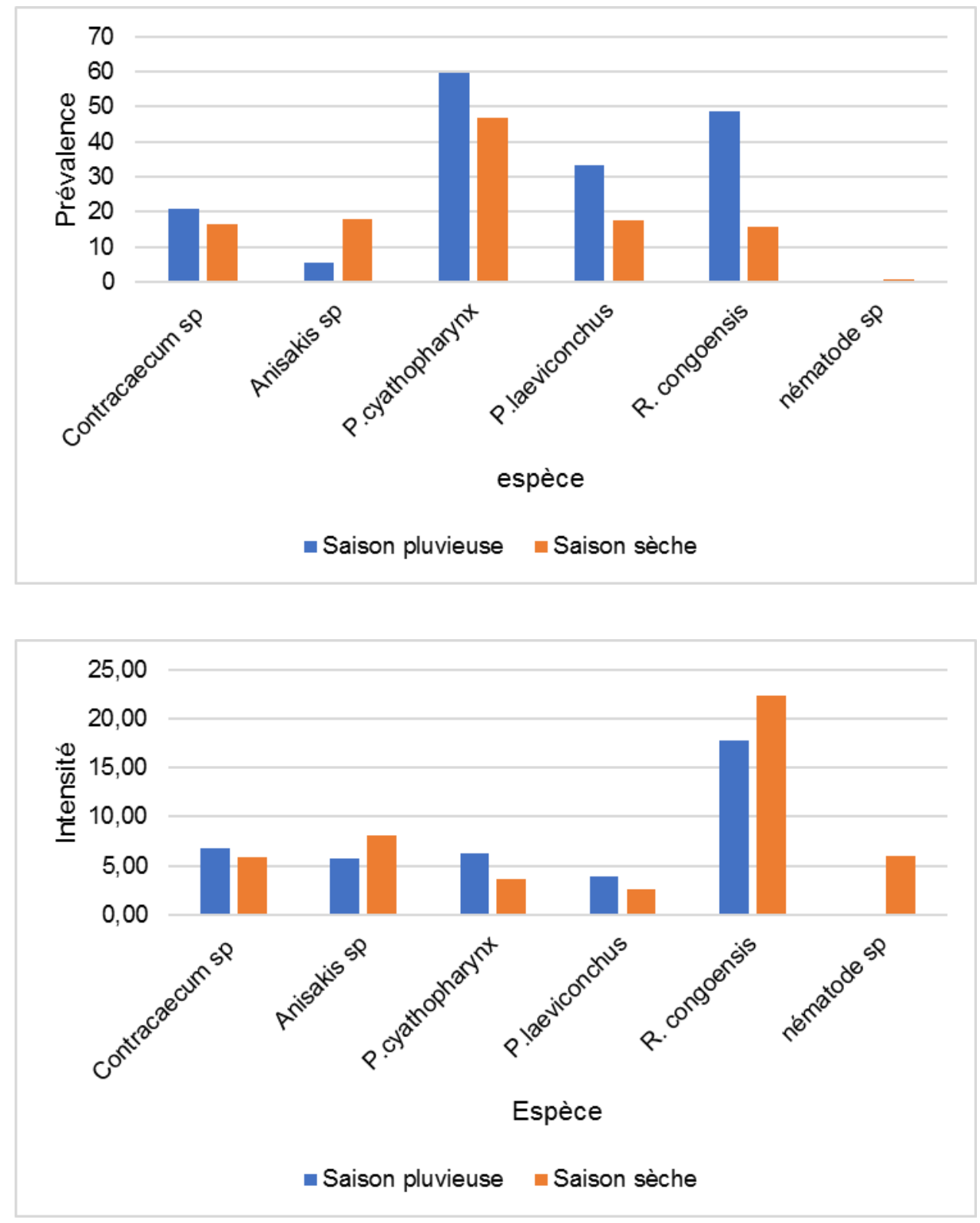

Figure 3 : Prévalence et intensité moyenne des espèces de nématodes en fonction des saisons. 

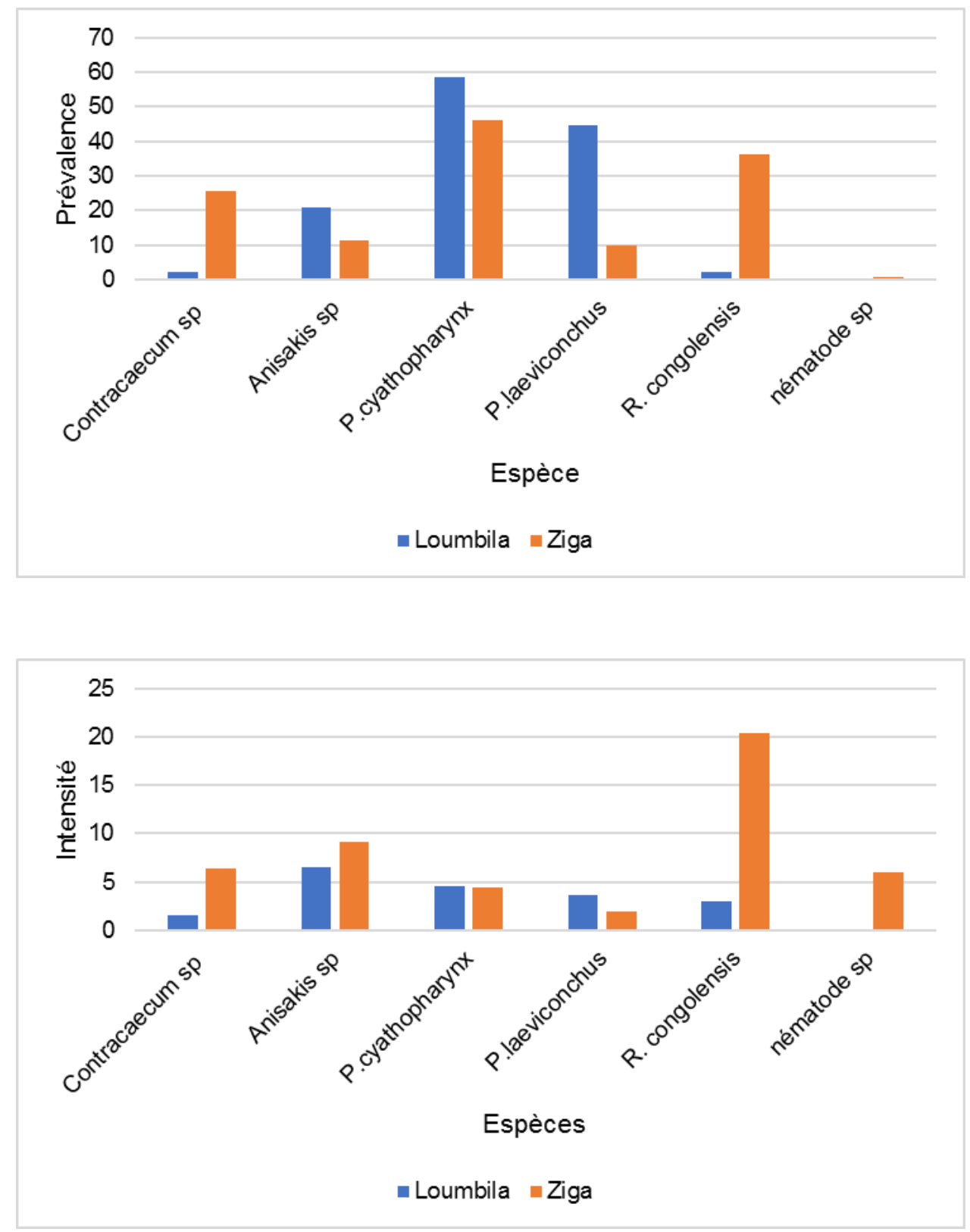

Figure 4 : Prévalence et intensité des espèces de nématodes en fonction des sites d'études.

\section{DISCUSSION}

\section{Diversité des nématodes}

Les nématodes sont fréquemment rencontrés chez les poissons du genre Clarias. Les espèces de nématodes telles que Paracamallanus cyathopharynx, Procamallanus laeviconchus, Contracaecum sp sont largement répandues chez Clarias spp et surtout chez ces deux espèces morphologiquement semblables, $C$. gariepinus et $C$. anguillaris (Nkwengulila and Mwita, 2004 ; Barson and Avenant-Oldewage, 2006; Olumuyiwa and Olatunde, 2014). Rhabdochona congolensis aussi, a été récolté chez les Clariidae (Mwita, 2014). Selon Paperna (1996), P. cyathopharynx et $P$. 
laeviconchus sont des parasites du genre Clarias. Ces genres sont distribués partout en Afrique où des recherches de nématodes parasites de poissons ont été réalisées. Les stades adultes des nématodes tel que Contracaecum évoluent chez les oiseaux piscivores. D'après Paperna (1996), les larves de nématodes apparaissent enkystées dans les tissus ou libres dans la cavité. Plusieurs larves sont souvent dans la cavité abdominale ou dans la cavité péricardiaque.

Nos investigations ont montré que $P$. cyathopharynx était plus élevé en nombre et en prévalence. Au Zimbabwe, Moyo et al. (2009) ont rapporté dans leur étude chez Clarias gariepinus que le nématode $P$. cyathopharynx et le cestode Tetracampos ciliotheca avaient les prévalences les plus élevées. $P$. cyathopharynx a déjà été décrit dans des études antérieures (Svitina et al., 2019). Il est un synonyme de $P$. senegalensis (Svitina et al., 2019). Il a déjà été observé au Burkina Faso (Kabré, 1997 ; Sinaré, 2009). P. cyathopharynx est largement distribué dans les plans d'eau du Burkina Faso et rencontrés dans beaucoup de poissons d'eau douce (Synodontis schall, Clarias anguillaris, Auchenoglanis occidentalis, Hepsetus odoe, Polypterus bichir) (Kabré, 1997). Rindoria et al. (2020) ont rapporté que les spécimens de $P$. cyathopharynx ont été trouvés dans l'intestin à côté du rectum.

Procamallanus laeviconchus est un parasite très largement répandu chez les poissons d'eau douce. Ce parasite généraliste (Okogwu et al., 2011) est présent presque chez toutes les familles des poissons d'eau douce. Il a déjà été rencontré au Burkina Faso chez plusieurs poissons (Kabré, 1997 ; Sinaré, 2009). Le rôle de l'hôte et de l'écosystème dans la distribution de P. laeviconchus a été déjà étudié (Okogwu et al., 2011). Nombreux sont les auteurs qui ont rencontré $P$. laeviconchus dans l'estomac (Barson and Avenant-Oldewage, 2006; Sorour and Hamouda, 2019) et dans l'intestin (Houénou Sèdogbo et al., 2019) comme dans la présente étude. P. laeviconchus est localisé généralement dans l'estomac. C'est donc un parasite spécifique de l'estomac des poissons même si on le rencontre quelque fois dans l'œsophage ou dans l'intestin. Noor El-Din et al. (2009), ont trouvé $P$. laeviconchus profondément pénétré dans le mucus de la paroi de l'estomac et de l'intestin de l'hôte et cela a causé des effets pathologiques graves. Le fait qu'il vit dans l'estomac prouve qu'il a une capacité de résistance à l'acidité de l'estomac.

Rhabdochona congolensis est moins étudié que Procamallanus laeviconchus, Paracamallanus cyathopharynx et Contracaecum sp. Il avait déjà été observé chez quelques poissons (Synodontis schall, Clarias anguilaris, Auchenoglanis occidentalis, Malapterurus electricus, Bagrus bajad, Alestes dentex, Schilbe sp, Tilapia zillii, Oreochromis niloticus et Brycinus nurse) du Burkina Faso (Sinaré, 2009 ; Sinaré et al., 2016). Les spécimens de $R$. congolensis ont été décrits pour la première fois au Congo (Campana-Rouget, 1961). Nous avons récolté $R$. congolensis au stade adulte et larvaire. Campana-Rouget (1961) a récolté également des larves et des adultes de Rhabdochona au Congo, mais le site de fixation des parasites chez l'hôte n'a pas été précisé.

Contracaecum sp que nous avons observé est le même que celui qui a été déjà décrit au Burkina Faso (Kabré, 1997) au stade générique et à l'état larvaire. Ces parasites étaient rencontrés chez $C$. anguillaris et Mormyrus rume au lac Tingrela et à Di (Kabré, 1997) dans les grandes eaux du Burkina Faso, là où il y a la possibilité de rencontrer plus d'hôtes définitifs (les cormorans, les hérons et autres oiseaux). Les études précédentes n'ont pas précisé la localisation du parasite chez l'hôte, mais dans notre étude, les spécimens de Contracaecum sp ont été observés dans la cavité générale, sur les mésentères et dans les parois du tube digestif. Yanong (2002) a trouvé également des espèces de Contracaecum sp dans plusieurs endroits de l'hôte dans la cavité générale, y compris le foie, les muscles, le cœur et la vessie. De nombreuses études ont montré que Contracaecum sp se retrouve chez les poissons sous forme de larves dans la cavité abdominale (Benant-Arson, 2004 ; 
Barson and Avenant-Oldewage, 2006 ; Gulelat et al., 2013). Pour l'identification spécifique du parasite, il faut nécessairement le parasite adulte chez l'hôte définitif afin d'avoir toutes les caractéristiques morphoanatomiques nécessaires ou une étude moléculaire pour l'identification.

Le nématode du genre Anisakis est un parasite fréquemment rencontré chez les poissons marins. En effet, de nombreuses études ont été réalisées chez les poissons de mer (Chou et al., 2011 ; Koinari et al., 2013 ; Oliva, 2013) où le nématode a été rencontré. Selon ces études, Anisakis (simplex) a été rencontré sous forme larvaire et généralement au stade L3 chez les poissons. Le parasite a trois hôtes dans son cycle de développement dont deux hôtes intermédiaires et un hôte définitif. Le poisson constitue le deuxième hôte intermédiaire. Les larves de stade 3 de Anisakis simplex ont été trouvées principalement dans l'intestin et la cavité cœlomique ; elles sont encapsulées dans la paroi viscérale, à la surface des organes ou dans les mésentères (Chaabouni et al., 2011). Dans la présente étude, nous avons récolté Anisakis sp dans la cavité, sur les mésentères, dans la paroi du tube digestif et sur le foie. La morphologie de nos individus, la présence de dents de pénétration, du ventricule œsophagien, des lèvres et du micron en plus de leur localisation chez l'hôte nous font penser que les spécimens que nous avons trouvés sont des espèces du genre Anisakis. C'est la première fois que cette espèce est rencontrée chez les poissons au Burkina Faso.

Contracaecum sp et Anisakis sp sont des agents de zoonose, transmis à l'homme par l'ingestion de chair de poissons ou de crustacés crus ou mal cuits. Des études ont montré que l'ingestion de la larve de Anisakis simplex peut causer une allergie en consommant l'allergène de ce parasite (Vidacek et al., 2009).

\section{Analyse de la dynamique et de la distribution des nématodes}

Les nématodes sont des parasites dont la plupart des adultes vivent dans la lumière du tube digestif et les larves dans la cavité générale de l'hôte ou dans les organes (Paperna, 1996). Dans notre investigation, le pourcentage d'infestation des nématodes a diminué au niveau de la dernière tranche d'âge (c'est-à-dire chez les grands poissons) car en ce moment les poissons seraient très matures et leur défense immunitaire serait bien développée. Une réponse immunologique pourrait réagir contre les parasites qui pourraient même disparaître. Les plus grands poissons sont moins infestés parce que leur système immunitaire est bien développé. Ils sont chargés car ils accumulent les parasites avec le temps, étant grands également ils peuvent contenir beaucoup de parasites. Cela peut être toujours expliqué par la diminution du contact ou du frottement entre les hôtes intermédiaires et les poissons. C. anguillaris est un poisson omnivore (N'Guessan et al., 2010) où les adultes consomment plus de poissons, d'insectes et d'autres macroinvertébrés; les juvéniles consomment le zooplancton y compris les copépodes et les détritus. Si les grands poissons réduisent leur consommation en crustacés qui sont généralement les hôtes intermédiaires cela contribue à diminuer leur contamination.

La fluctuation du nombre des parasites en fonction des saisons peut être due à l'abondance relative des hôtes intermédiaires en fonction des saisons. Selon Monney et al. (2015), le zooplancton est plus diversifié en saison des pluies et plus abondant en saison sèche. Nos résultats montrent que la prévalence des différentes espèces de nématodes est plus haute en saison pluvieuse. L'occurrence et l'incidence des parasites en saison pluvieuse sont dues probablement à l'abondance des hôtes intermédiaires en saison pluvieuse. La prédominance de la prévalence, de l'intensité et du nombre de certaines espèces de nématodes en saison sèche peut être expliquée par le fait que le niveau d'eau ayant diminué, le volume devient faible et les différents hôtes sont plus en contact.

Parmi les espèces de nématodes étudiées, seule $P$. laeviconchus est plus prédominante en prévalence et en intensité dans le lac de barrage de Loumbila. Cette 
différence particulière de cette espèce pourrait être liée à des paramètres intrinsèques propres à ces individus et aux conditions du milieu (nature, état...).

\section{Conclusion}

Cette étude nous a permis d'inventorier 06 espèces de nématodes chez Clarias anguillaris dont une (Anisakis sp.) qui est pour la première fois rencontrée au Burkina Faso chez les poissons. Deux espèces sont d'importances zoonotiques : Contracaecum $\mathrm{sp}$ et Anisakis sp. Presque tous les organes internes de l'hôte sont des sites de fixation des nématodes. Une étude supplémentaire serait donc nécessaire sur les hôtes définitifs (oiseaux aquatiques et piscivores) pour pouvoir déterminer spécifiquement les espèces rencontrées au stade larvaire.

\section{CONFLIT D'INTERETS}

Les auteurs déclarent qu'il n'y a aucun conflit d'intérêt concernant ce manuscrit.

\section{CONTRIBUTIONS DES AUTEURS}

YS a participé à la conception de la thématique, l'analyse des données, l'interprétation des données, la rédaction de l'article et la correction de l'article. MB a participé à la conception de la thématique, l'interprétation des données, la lecture et la correction de l'article. MNK a participé à la lecture et à la correction de l'article. GBK a participé à la conception de la thématique, la lecture et la correction de l'article.

\section{REMERCIEMENTS}

Nous remercions les membres du laboratoire de Biologie et Ecologie Animales de l'Université Joseph- Ki-Zerbo qui ont contribué à la réalisation de ce travail. Nous remercions également l'Agence Universitaire de la Francophonie (AUF) pour leur soutien matériel et financier.

\section{RÉFÉRENCES}

Akinsanya B, Otubanjo OA. 2006. Helminth Parasites of Clarias gariepinus (Clariidae) in Lekki Lagoon, Lagos,
Nigeria. Rev. Biol. Trop., 54(1): 93-99. DOI: $10.15517 /$ rbt.v54i1.14003

Ayanda IO. 2008. Comparison of parasitic helminth infection between the different age groups of Clarias gariepinus from Asa dam Ilorin, north-central Nigeria. African Journal of Environmental Science and Technology, 2(11): 404-406. http://www.academicjournals.org/AJest

Barson M. 2004. The occurrence of Contracaecum sp . larvae (Nematoda: Anisakidae) in the catfish Clarias gariepinus (Burchell) from Lake Chivero, Zimbabwe. Onderstepoort Journal of Veterinary Research, 71: 3539. DOI: $10.4102 /$ ojvr.v71i1.283

Barson M, Avenant-Oldewage A. 2006. Nematode parasites of Clarias gariepinus (Burchell, 1822) from the Rietvlei Dam, South Africa. The Onderstepoort Journal of Veterinary Research, 73(2): 87-94. DOI: 10.4102/ojvr.v73i2.152

Campana-Rouget Y. 1961. Nématodes de Poissons ( $3^{\text {eme }}$ edn). Editions Edouard et Albert : Paris.

Chaabouni M, Dhaouadi R, El Fekih O. 2011. Les parasites des produits de la mer. Les Services Vétérinaires Bulletin d'Information, 10 : 1-18.

Chong R, Bousfield B, Brown R. 2011. Fish Disease Management. Disease Management, 1(8): 1-12.

Chou YY, Wang CS, Chen HG, Chen HY, Chen SN, Shih HH. 2011. Parasitism between Anisakis simplex (Nematoda: Anisakidae) third-stage larvae and the spotted mackerel Scomber australasicus with regard to the application of stock identification. Veterinary Parasitology, 177(3-4): 324-331. DOI: 10.1016/j.vetpar.2010.12.003

Compaoré I, Toguyeni A, Rougeot C, Kestemont P, Melard C. 2016. Etude comparative des performances zootechniques d'une souche domestiquée de Clarias gariepinus (Burchell, 1822) et d'une souche sauvage de Clarias anguillaris (Linnaeus, 1758). Int. J. Biol. 
Chem. Sci., 10(5): 2138-2150. DOI: http://dx.doi.org/10.4314/ijbcs.v10i5.16

FAO/WHO [Food and Agriculture Organization of the United Nations/World Health Organization]. 2014. Multicriteria-based ranking for risk management of food-borne parasites. FAO/WHO Microbiological Risk Assessment Series 23, Rome, 302 p.

Gibbons LM. 2010. Keys to the Nematode Parasites of Vertebrate. Cambridge University Press: Cambridge.

Gulelat Y, Yimer E, Asmare K, Bekele J. 2013. Study on parasitic helminths infecting three fish species from koka reservoir, Ethiopia. Ethiop. J. Sci., 36(2): 73-80.

Houénou Sèdogbo M, Zannou BT, Siko JE, Tossavi ND, Togla I, Fiogbé ED, Ibikounlé M. 2019. Faune des métazoaires parasites de Clarias gariepinus Clariidae) et de Oreochromis niloticus (Cichlidae), deux poissons des whédos du delta supérieur du fleuve Ouémé au sud du Bénin. Int. J. Biol. Chem. Sci., 13(2): 983-997. DOI: https://dx.doi.org/10.4314/ijbcs.v13i2.33

Kabré BG. 1997. Parasites des poissons du Burkina Faso: Faunistique, ultrastructure, biologie. Thèse d'Etat, Université de Ouagadougou, Burkina Faso, $308 \mathrm{p}$.

Koinari M, Karl S, Elliot A, Ryan U, Lymbery AJ. 2013. Identification of Anisakis species (Nematoda: Anisakidae) in marine fish hosts from Papua New Guinea. Veterinary Parasitology, 193: 126-133. DOI: http://dx.doi.org/10.1016/j.vetpar.2012.1 2.008

Kuchta R, Burianova A, Jirku M, de Chambrier A, Oros M, Brabec J, Scholz T. 2012. Bothriocephalidean tapeworms (Cestoda) of freshwater fish in Africa, including erection of of Kirstenella $\mathrm{n}$. gen. and description of Tetracampos martinae n. sp. Zootaxa., 35: 1-35. DOI: https://doi.org/10.11646/zootaxa.3309.1. 1
Mclaughlin J, Concordia D. 2008. Protocoles du réseau d'évaluation et de surveillance écologiques (RÉSÉ) pour mesurer la biodiversité : parasites des oiseaux. Société Canadienne de Zoologie, 1-95.

Monney IA, Etile RN, Ouattara IN, Kone T. 2015. Seasonal distribution of zooplankton in the Aby-Tendo-Ehy lagoons system (Côte d'Ivoire, West Africa). Int. J. Biol. Chem. Sci., 9(5): 2362-2376.

DOI: http://dx.doi.org/10.4314/ijbcs.v9i5.9

Moyo DZ, Chimbira C, Yalala P. 2009. Observations on the Helminth Parasites of Fish in Insukamini Dam, Zimbabwe. Research Journal of Agriculture and Biological Sciences, 5(5): 782-785. http://www.insipub.com/.../782-785

Mwita CJ. 2014. Metazoan parasites of Clariid fishes, Lake Victoria: reflection of the original fauna in the lake? Natural Science, 6: 651-658. DOI: http://dx.doi.org/10.4236/ns.2014.69064

N'guessan SY, Doumbia L, N'Goran GK, Gourène G. 2010. Habitudes Alimentaires du Poisson-chat, Clarias Anguillaris (Linné, 1758) (Clariidae) dans un Hydrosystème Fluvio-lacustre Ouest- Africain (Rivière Bia, Côte d'Ivoire). European Journal of Scientific Research, 46(2): 275-285.

Nkwengulila G, Mwita C. 2004. Spatial disribution of parasites along the gut of the catfish Clarias gariepinus (Burchell, 1822) (Clariidae) from the Mwanza gulf, lake Victoria. Tanzania Journal of Science, 30(1): 63-70. DOI: 10.4314/tjs.v30i1.18388

Noor El-Din SNEA, Khalil AI, El-Sheekh HE, Radwan NA. 2009. Histopathological effect of the spiruoid nematode Procamallanus laeviconchus in the stomach and intestine of nile cat fish Clarias gariepinus. Egypt. J. Exp. Biol. Zool., 5: 109 -113.

Okogwu OI, Ani CO, Uneke IB. 2011. Distribution of Procamallanus laevionchus in relation to environmental variables in three tropical african rivers : the role of the host and ecosystem. Acta 
Zoologica, 21(1992): 145-152. DOI: 10.2478/v10043-011-0015-1

Oliva ME. 2013. Is Anisakis simplex s.l. a biological marker for stock identification of Strangomera bentincki from Chile? Journal of Fish Biology, 83: 412-416. DOI: $10.1111 / \mathrm{jfb} .12176$

Olumuyiwa O, Olatunde O. 2014. Multiple infections of Helminths in the alimentary system of Clarias gariepinus (Burchell, 1822) in a tropical reservoir. International Journal of Fisheries and Aquaculture, 6(6): 62-70. DOI: 10.5897/IJFA2014.0417

Paperna I. 1996. Parasites, infections and diseases of fishes in Africa: an update. FAO/CIFA Technical Paper, Rome, 220 p.

Rindoria NM, Santos QMD, AvenantOidewage A. 2020. Additional morphological features and molecular data of Paracamallanus cyathopharynx (Nematoda: Camallanidae) infecting Clarias gariepinus (Actinopterygii: Clariidae) in Kenya. Journal of Parasitology, 106(1): 157-166. DOI: 10.1645/19-62

Sinare Y. 2009. Les Helminthes chez les poissons d'intérêt économique au Burkina Faso : Biodiversité. Mémoire de Diplôme d'Etudes Approfondies (DEA), Université de Ouagadougou, Ouagadougou, $72 \mathrm{p}$.

Sinaré Y, Boungou M, Ouéda A, Mano K, Kpoda WN, Sakiti NG, Kabré GB. 2015. Occurrence of Tetracampos ciliotheca (Cestoda: Bothriocephalidea) in the gall bladder of Clarias anguillaris in Burkina Faso. African Journal of Aquatic Science, 40(4): 433-437. DOI: http://dx.doi.org/10.2989/16085914.2015 .1082900

Sinaré Y, Boungou M, Ouéda A, Gnémé A, Kabré GB. 2016. Diversity and seasonal distribution of parasites of Oreochromis niloticus in semi-arid reservoirs (West
Africa, Burkina Faso). African Journal of Agricultural Research, 11(13): 11641170. DOI: 10.5897/AJAR2015.10408

Sinaré Y. 2017. Diversité et dynamique des Métazoaires parasites de Clarias anguillaris (Linnaeus, 1758) et de Oreochromis niloticus (Linnaeus, 1758) au Burkina Faso. Thèse unique, Université Joseph Ki-Zerbo, Ouagadougou, $162 \mathrm{p}$.

Sorour SHS, Hamouda AH. 2019. Prevalence of nematodes infestation in Clarias gariepinus from El-Burullus Lake and Lake Nasser, Egypt. Iraqi Journal of Veterinary Sciences, 33(2): 181-188. DOI: 10.33899/ijvs.2019.162963

Svitina R, Truterd M, Kudlaif O, Smitd NJ, du Preez L. 2019. Novel information on the morphology, phylogeny and distribution of camallanid nematodes from marine and freshwater hosts in South Africa, including the description of Camallanus sodwanaensis n. sp. International Journal for Parasitology, Parasites and Wildlife, 10: 263-273. DOI: 10.1016/j.ijppaw.2019.09.007

Usip LPE, Udoidiong OM, Ekwu A, Ekpo I, Essienibok M. 2010. Parasites spectrum of cichlid species in the lower. Science and Technology, 2(1): 59-68.

Vidacek S, de las Heras C, Solas MT, Mendiz'abal A, Rodriguez-Mahillo AI, Gonzalez-Munoz M, Tejada M. 2009. Anisakis simplex allergens remain active after conventional or microwave heating and pepsin treatments of chilled and frozen L3 larvae. Journal of the Science of Food and Agriculture, 89: 1997-2002. DOI: $10.1002 /$ jsfa.3677

Yamaguti S. 1961. Systema helminthum: The Nematode of Vertebrates Part 1. Interscience Publishers Inc.: New York.

Yanong RPE. 2002. Nematode (Roundworm) Infections in Fish. Dean. Circular, 91:19. 